\title{
Quality of Clinical Evaluation from Viewpoint of Nurse Interns and Nursing Unit Clerks; Nursing Students of the School of Nursing and Midwifery
}

\author{
Zahra Riahi Roohi and Shayesteh Salehi* \\ Faculty of Nursing and Midwifery, Isfahan (Khorasgan) Branch, Islamic Azad University, Isfahan, Iran; \\ Salehi@nm.mui.ac.ir
}

\begin{abstract}
Quality of nurse training programs requires clinical evaluation. The purpose of this study was to achieve a perspective of realities from viewpoint of nursing students during their internship and clerkship. This study was descriptive and quantitative; 180 nurse inters and nursing unit clerks were enrolled for the study. Data was collected using a researcher made questionnaire. Content validity of the questionnaire was confirmed by a group of faculty members and clinical professors; reliability of the questionnaire was confirmed by Cronbach's alpha (0.89). The questionnaire consisted of two parts; demographic data and data related to clinical evaluation in four areas including content of evaluation, the role of instructor in evaluation, evaluation process and outcome of evaluation. The questionnaire was scored on a five-point Likert scale from always to never. At the end, there were two open questions about suggestions on clinical evaluation. Data were analyzed by SPSS22, descriptive and analytic statistics. Results showed that nurse interns evaluated the instructor as good ( $4.05 \pm 0.06)$, evaluation content as average $(3.65 \pm 0.06)$, evaluation process as average $(2.77 \pm 0.76)$ and outcome of evaluation as poor $(2.41 \pm 0.69)$. Nursing unit clerks evaluated the instructor as good ( $3.84 \pm 0.59$ ), evaluation content as average (3.51 \pm 0.68$)$, evaluation process as average $(2.60 \pm 0.78)$ and outcome of evaluation as poor ( $2.56 \pm 0.63)$. According to the results, quality of clinical evaluation of nursing students requires a review in existing processes and tools, as well as revision in programs and clinical evaluations by planners and instructors.
\end{abstract}

Keywords: Clinical Evaluation, Nurse Intern, Nursing Unit Clerk

\section{Introduction}

Nursing is a profession which involves different situations from health to illness. Nursing profession focuses on health promotion and helps families and groups to meet their medical needs. Due to the complex nature of nursing profession and variety of medical situations experienced by nurses, society requires experienced and skilled nurses to deliver health care services ${ }^{1}$. Nursing profession is a mixture of science and art, requiring theoretical and practical knowledge. In order to train a qualified nurse, nursing schools need to use effective strategies for promoting knowledge, skills and attitudes of students to enable them to combine theory and practice effectively ${ }^{2}$. Three core processes of nursing curriculum are design, implementation and evaluation. Setting and evaluating educational goals are important stages of design process ${ }^{3}$. Evaluation process forms an essential part of clinical education. Evaluation process is important because it depicts the effectiveness of educational programs and ensures efficient design and implementation of educational programs. Various studies conducted on nursing education have focused on evaluation to determine the extent to which evaluation is accurate ${ }^{4}$. Evaluation is the essential part of a program and one of the most important aspects of human resource management; some consider evaluation as vulnerable point of management, because only it can help to address and eliminate shortcomings of a program ${ }^{5}$. Since clinical training is a process in which the student acquires clinical experiences with patients and hospital environment, it is very difficult to evaluate and judge clinical skills acquired ${ }^{6}$. Effective evaluation process

${ }^{*}$ Author for correspondence 
can attract the qualified students. Hence, the educational system is expected to prepare students for care in complex situations and emphasize the knowledge, attitudes and skills required ${ }^{7}$. Evaluation is able to distinguish between students, cover goals and assess all educational areas. The goals and outcomes of evaluation include: 1 . To maximize abilities of learners through guidance and motivation for future learning; 2. Screen inefficient and unqualified learners; 3. To develop a basis for selection of qualified people to enter higher educational levels; 4 . To find out educational problems of students and plan to eliminate them ${ }^{8}$. Evaluation gives value to the data collected by one or more measurements and involves judgment on strengths and weaknesses of different aspects of a phenomenon. Evaluation is a step in the review process which measures quality based on standards of performance. Educational evaluation reviews the data related to measurement of total outcomes of teaching-learning processes. Clinical evaluation provides data for judgment on achievement of learning outcomes by nursing students. Clinical evaluation judges skills of students in relation to patient care standards. The final outcome of clinical evaluation is to ensure high quality and safe care ${ }^{9}$. Given the significant role of clinical evaluation in nurse training courses, the present study examines viewpoints of nurse interns and nursing unit clerks about clinical evaluation to identify strengths and weaknesses of clinical education in order to improve quality of clinical evaluation of nursing students.

\section{Materials and Methods}

This descriptive cross-sectional study (which is part of a larger study) was conducted in 2015 to obtain view points of nursing students regarding clinical evaluation. Participants included 180 nurse interns and nursing unit clerks during 2014-2015 from the School of Nursing and Midwifery, Islamic Azad University, Khorasgan of Isfahan. Census was used for sampling. The study was conducted in all clinical environments in which nurse interns and nursing unit clerks worked. The data was collected by a researcher-made questionnaire in three parts. The first part included demographic data (age, gender, marital status, educational status and occupational status); the second part contained 33 items in four areas including evaluation content (8 items), the role of instructor (10 items), evaluation process ( 9 items) and evaluation outcome (6 items). Since clinical evaluation is a process, items might overlap. The items were scored on a5-point Likert scale. Subjects were asked to fill the questionnaire according to their experiences of clinical evaluations conducted during academic years. Then, responses were calculated and scored. The third part consisted of two open questions about comments and suggestions on clinical evaluation. Content validity was used to determine scientific validity of the questionnaire. The questionnaire was drafted by reviewing numerous clinical studies, other archival studies and feedbacks of clinical professors. Then, the draft was given to a group of faculty members and clinical instructors to make modifications, if necessary, for the final questionnaire. In order to determine reliability of the questionnaire, Cronbach's alpha was used to measure internal consistency; the Cronbach's alpha was estimated at 0.89 . Data was described by descriptive statistics and analyzed by independent t-test. The software SPSS22 was used for data analysis.

This project was approved by the ethics committee of the University of Medical Sciences (code IR.MUI. REC.1394.4.26) on July 17, 2015. In order to comply with other ethical considerations, written consent (I have read and consent to participate in the study voluntarily) was obtained from subjects; objectives, inclusion criteria and advantages of participation were explained for the subjects. The subjects were ensured of privacy protection and voluntary participation. The questionnaires were selfadministered by the subjects.

\section{Results}

Participants included 180 nursing students $(51.7 \%$ nurse interns; mean age $22.16 \pm 2.170)$ and $(48.3 \%$ nursing unit clerks; mean age $23.21 \pm 1.741)$. Nurse interns were both male (9.7\%) and female (90.3\%), while all nursing unit clerks were female. Tables 1-4 list viewpoints of students regarding clinical evaluation. Table 5 presents conclusions of the study.

Table 1. Distribution of subjects based on content of evaluation

\begin{tabular}{lcccc}
\hline \multirow{2}{*}{ Evaluation content } & \multicolumn{2}{c}{ Interns } & \multicolumn{2}{c}{ Clerks } \\
\cline { 2 - 5 } & No. & $\%$ & No. & $\%$ \\
\hline Poor (8-18 points) & 2 & 2.2 & 4 & 4.6 \\
Average (19-29 points) & 47 & 50.5 & 48 & 55.2 \\
Good (30-40 points) & 44 & 47.3 & 35 & 40.2 \\
\hline Total & $\mathbf{9 3}$ & $\mathbf{1 0 0 . 0}$ & $\mathbf{8 7}$ & $\mathbf{1 0 0 . 0}$ \\
\hline
\end{tabular}


In relation to quality of evaluation content, the highest frequency was related to 'Assignments of the wards are effective on the evaluation score. The lowest frequency was related to 'Clinical evaluation is based on the goals achieved in the course' and 'Evaluation is performed clearly and explicitly'. The quality of evaluation content was evaluated as average.

Table 2. Distribution of subjects based on the role of instructor

\begin{tabular}{lcccc}
\hline Instructor & \multicolumn{2}{c}{ Intern } & \multicolumn{2}{c}{ Clerk } \\
\cline { 2 - 5 } & No. & $\%$ & No. & $\%$ \\
\hline Poor (10-23 points) & 1 & 1.1 & 0 & 0.0 \\
Average (24-37 points) & 29 & 31.2 & 37 & 42.5 \\
Good (38-50 points) & 63 & 67.7 & 50 & 57.5 \\
\hline Total & $\mathbf{9 3}$ & $\mathbf{1 0 0 . 0}$ & $\mathbf{8 7}$ & $\mathbf{1 0 0 . 0}$ \\
\hline
\end{tabular}

In relation to the role of instructor in clinical evaluation, the highest frequency was related to 'Sufficient clinical skill of instructor is effective in clinical evaluation' and the lowest frequency was related to 'Evaluation and scoring is done in the presence of students. The role of instructor in clinical evaluation was evaluated as good.

Table 3. Distribution of subjects based on evaluation process

\begin{tabular}{lcccc}
\hline Evaluation process & \multicolumn{2}{c}{ Intern } & \multicolumn{2}{c}{ Clerk } \\
\cline { 2 - 5 } & No. & $\%$ & No. & $\%$ \\
\cline { 3 - 5 } Poor (9-21 points) & 32 & 34.4 & 33 & 37.9 \\
Average (22-33 points) & 50 & 53.8 & 47 & 54.0 \\
Good (34-45 points) & 11 & 11.8 & 7 & 8.0 \\
\hline Total & $\mathbf{9 3}$ & $\mathbf{1 0 0 . 0}$ & $\mathbf{8 7}$ & $\mathbf{1 0 0 . 0}$ \\
\hline
\end{tabular}

In relation to quality of evaluation process, the highest frequency was related to 'Ethics and professional conduct of students are considered in clinical evaluation' and the lowest frequency was related to 'Self-evaluation forms are distributed among students'. Students evaluated the quality of evaluation process as average.

Table 4. Distribution of subjects based on evaluation outcome

\begin{tabular}{lcccc}
\hline Evaluation outcome & \multicolumn{2}{c}{ Intern } & \multicolumn{2}{c}{ Clerk } \\
\cline { 2 - 5 } & No. & $\%$ & No. & $\%$ \\
\hline Poor (6-13 points) & 37 & 39.8 & 27 & 31.0 \\
Average (14-21 points) & 54 & 58.1 & 53 & 60.9 \\
Good (22-30 points) & 2 & 2,2 & 7 & 8.0 \\
\hline Total & $\mathbf{9 3}$ & $\mathbf{1 0 0 . 0}$ & $\mathbf{8 7}$ & $\mathbf{1 0 0 . 0}$ \\
\hline
\end{tabular}

In relation to quality of evaluation outcomes, the highest frequency was related to 'Clinical evaluation do not cause stress and anxiety among students' and the lowest frequency was related to 'Clinical evaluation is based on accurate and objective criteria in an uniform pattern'. Quality of evaluation outcome was evaluated as average.

Table 5. Comparison of mean scores of evaluation given by subjects

\begin{tabular}{lcccccc}
\hline \multirow{2}{*}{ Course } & \multicolumn{3}{c}{ Internship } & \multicolumn{3}{c}{ Clerkship } \\
\cline { 2 - 8 } Evaluation areas & Mean & SD & Rank & Mean & SD & Rank \\
\hline Evaluation content & 3.65 & 0.60 & 2 & 3.51 & 0.68 & 2 \\
Role of instructor & 4.05 & 0.60 & 1 & 3.84 & 0.59 & 1 \\
Evaluation process & 2.77 & 0.76 & 3 & 2.60 & 0.78 & 3 \\
Evaluation outcome & 2.41 & 0.69 & 4 & 2.56 & 0.63 & 4 \\
\hline
\end{tabular}

Comparing four areas of clinical evaluation, students gave the lowest score to evaluation outcome and the highest score to the role of instructor.

\section{Discussion}

According to nursing students, the role of instructor obtained the highest score for quality. Khosravi et al. reported that instructor needs the required skills, and updated information on the ward and relevant internship to train clinical skills and evaluates students ${ }^{10}$. In another study, clinical professor was considered as the most important person, particularly in the clinical evaluation. Students believed that clinical professor needs skills and updated information to teach and evaluate ${ }^{11}$.

According to nursing students, evaluation content obtained the second high score for quality. Written assignments had the highest frequency in clinical evaluation. In a study, written assignments were reported as a suitable tool for clinical evaluation ${ }^{12}$.

Evaluation content was followed by evaluation process in terms of the score obtained for quality. Discarded selfevaluation forms had the highest frequency. In a study, self-evaluation was reported as one of the methods used to determine knowledge and clinical skills. The evaluated students should have an active role in their evaluation to understand their responsibilities, strengths and weaknesses ${ }^{13}$. In another study, self-evaluation is reported as one of the helpful methods to determine knowledge and clinical skills, because a learner is a suitable source 
of information about that learner and no one, except the learner, does have a clear vision of him ${ }^{14}$.

Evaluation outcome obtained the lowest score for quality. The most frequent item was related to the lack of accurate and objective criteria. This result is consistent with a study in which stereotypical evaluation and incoherent evaluation were reported as challenges of clinical evaluation ${ }^{15}$. However, objective evaluation and the fact that subjectivity of the observer will influence the evaluation have been previously raised. Equality, stability and fairness are important discussions which are often ignored in clinical evaluation ${ }^{12}$.

In reviewing the results of open-ended questions which were responded by some of the subjects, following results are derived:

Some students believed that internship did not influence their learning performance; they believed that clinical evaluation was a formality. This is consistent with Borazbardanjani ${ }^{16}$. One student claimed that the performance of all students was the same in ward, while the scores were different. A study also noted that student performance was not proportionate to the given scores ${ }^{17}$. However, another student claimed that the scores were the same for students with good and poor performances; this is also consistent with another study ${ }^{18}$. Some of the students stated that quality of evaluation would be higher if clinical instructor and theoretical instructor was the same person. This is consistent with Delaram et al. ${ }^{19}$ who believed that it is helpful to use the same person for both theoretical and practical instructors. Some students stated that clinical conferences and nursing process in the presence of a real patient are suitable for evaluation. Common methods of conference, lecture or presentation of cases which are not seen in wardsare ineffective in evaluation. Khosravi ${ }^{10}$ also suggested presentation for improving quality of evaluation. Some students complained about the lack of coordination of instructors. They believed that some instructors are stricter in evaluation, while others are easy going. This is consistent with the finding that there are differences between clinical instructors; different instructors have different expectations and standards in clinical evaluation of students ${ }^{20}$.

\section{Conclusions}

According to the results, it seems that current evaluation system lacks a valid instrument; a specific model can enhance the performance of evaluation system. Improvement of content and process of evaluation can provide skilled human resources. Multidimensional evaluation and self-evaluation require specific facilities. The results suggest the need for interventions to change training programs with an emphasis on creative forms of evaluation.

\section{Acknowledgement}

This project is part of the report for master's degree in nursing education, Department of Nursing and Midwifery of Islamic Azad University, Khorasgan Branch, Isfahan. The author appreciates the clinical professors and instructors and students for participation in this study.

\section{References}

1. Mirzabeygi G, Sanjari S, Heidari S, Salemi S. Undergraduate education from viewpoints of nursing professors and students. Nursing Research. 2011; 20(6):64-74.

2. Khan BA, Fauziya A, Vazir N. Students' perceptions of clinical teaching and learning strategies: A Pakistani perspective. Nurse Education Today. 2012; 32(1):85-90.

3. Hasan-Zahraei R, Atash Sokhan G, Salehi S, Ehsanpour S, Hasanzadeh A. Comparison of the factors related to effective clinical training from viewpoint of instructors and students. Medical Education. 2007; 7(2):249-56.

4. Duers LE, Brown N. An exploration of student nurses' experiences of formative assessment. Nurse Education Today. 2009; 29(6):654-9.

5. Khadem-Hoseyni SM, Mokhtari-Nouri J, Feyzi F. Comparison of performance evaluation methods for nursing students in clinical education. Educational Strategies. 2012; 5(3):171-5.

6. Yanhun C, Watson R. A review of clinical competances assessment in nursing. Nurse Education Today. 2011; 31(3):1832-6.

7. Shipman D, Roa M, Hobten J, Wang Z. Using the analytic rubric an assessment in nursing education: the Positive and the negative. Nurse Education Today. 2012; 32(3):2469.

8. Joyce, B. Developing an assessment system: Facilitator's guide. Accreditation Council Grad Med Educ. 2006; 1:15-7.

9. Billings D, Halstead J. Teaching in nursing. 4th ed. St. Louis: Elsevier; 2012.

10. Khosravi S, Pazargardi M, Ashktorab T. Challenges of clinical evaluation of nursing students: A qualitative study. Education in Medical Sciences. 2011; 11(7):735-49.

11. Asfour HI, EL-Soussi AH. A qualitative study of effectiveness of clinical teaching. Journal of American Science. 2011; 7(7):409-20.

12. McCutchan JA. The experience of baccalaureate degree 
seeking nursing students undergoing the process of clinical evaluation appraisal [Dissertation ]. Indiana State University; 2010.

13. Wildeman N. A study of clinical performance by diploma nursing [Dissertation]. Canada: The University of Regina; 1989.

14. Belar C, Brown RA, Hersch LE, Hornyak LM, Rozensky $\mathrm{RH}$, Sheridan EP. Self-assessment in clinical health psychology: A model for ethical expansion of practice. Prof Psychol Res Pr. 2001; 32(2):135-41.

15. Sabzevari S, Abaszade A, Borhani F. Perceptions of Nursing instructors of challenges of clinical evaluation for nursing students: A qualitative study. Research and Development of Medical Sciences. 2013; 3(10):267-79.

16. Borazbardanjani S, Fereydoni-Moghadam M, Lourizadeh MR. Quality of clinical evaluation from viewpoint of students. Research and Development of Medical Sciences. 2008; 5(2):102-12.

17. Alavi M, Irajpour AR. Clinical evaluation of nursing students. Isfahan University of Medical Sciences, 2013; 13(10):796-808.

18. Kamran A, Sharafi A, Biria M, Dadkhah M. Quality of solutions for improvement of clinical training for nurses. Nursing Research. 2012; 27(7):25-31.

19. Delaram M, Raeisi Z, Alidousti M. Strengths and weaknesses of clinical training from viewpoint of nursing and widwifery students. Qom Medical Sciences. 2012; 6(2):7681.

20. Elcigil A, Yildirim H. Determining problems experienced by student nurses in their work with clinical educators in Turkey. Nurse Education Today. 2007; 27(13):491-8. 